\title{
Pattern Association for character recognition by Back-Propagation algorithm using Neural Network approach
}

\author{
Prof. S.P.Kosbatwar \\ Asst. Prof. \\ Prof.S.K.Pathan \\ Asst. Prof. \\ Dept.of Computer Engg \\ shyamkosbatwar@gmail.com \\ Dept. of Computer Engg. \\ Smt.Kashibai Navale College of Engineering,Vadgaon (bk),Pune -41
}

\begin{abstract}
The use of artificial neural network in applications can dramatically simplify the code and improve quality of recognition while achieving good performance. Another benefit of using neural network in application is extensibility of the system - ability to recognize more character sets than initially defined. Most of traditional systems are not extensible enough. In this paper recognition of characters is possible by using neural network back propagation algorithm.
\end{abstract}

\section{What is neural network}

Neural network are simplified models of the biological nervous system and therefore have drawn their motivation from the kind of computing performed by a human brain. An NN in general is a highly interconnected of a large number of processing elements called neurons in an architecture inspired by the brain. An NN can be massively parallel and therefore is said to exhibit parallel distributed processing. Neural Network exhibits characteristics such as mapping capabilities or pattern association, generalization, robustness, fault tolerance, and parallel and high speed information processing. Neural network learn by example. They can therefore be trained with known examples of a problem to acquire knowledge about it. Once appropriate trained the network can be put to effective use in solving 'unknown' or 'untrained' instances of the problem. Neural network adopt various learning mechanism of which supervised learning and unsupervised learning methods have turned out to be very popular. In supervised learning, a teacher is assumed to be present during the learning process, i.e. the network aims to minimize he error between target (desired) output presented by the teacher and the computed output to achieve better performance. However, in unsupervised learning, there is no teacher present to hand over the desired output and the network therefore tries to learn by itself, organizing the input instances of the problem.NN Architecture has been broadly classified as single layer feed forward networks, multilayer feed forward networks and recurrent networks, over the year several other NN.Architecture have evolved some of the well known NN system include backpropogation network, perceptron, ADALINE ,Boltzmann machine ,adaptive resonance theory, Self-organized feature map, and Hopfield network. Neural Network has been successfully applied to problem in the field of pattern recognition, image processing, data compression, forecasting and optimization to quote a few. 


\section{Backpropagation algorithm}

The architecture of the neural network is the one of a basically backpropagation network with only one hidden layer (although it is the same techniques with more layers). The input layer is constituted of 35 neuron (one per input pixel in the matrix, of course)., they are 8 hidden neurons, and 26 output neurons(one per letter) in this problem domain of character recognition. The weight matrix gives the weight factor for each input of each neuron. These matrices are what we can call the memory of the neural network. The learning process is done by adjusting these weight so that for each given input the output is as near as possible of a wanted output (Here the full activation of the output neuron corresponding to the character to be recognized) [1].

The training patterns are applied in some random order one by one, and the weights are adjusted using the backpropagation learning law. Each application of the training set patterns is called a cycle. The patterns have to be applied for several training cycles to obtain the output error to an acceptable low value. Once the network is trained, it can be used to recall the appropriate pattern for a new input pattern. The computation for recall is straightforward, in the sense that the weights and the output functions of the units in different layers are used to compute the activation values and the output signals. The signals from the output layer correspond to the output[2].

Backpropagation learning emerged as the most significant result in the field of artificial neural networks. The backpropagation learning involves propagation of the error backwards from the output layer to the hidden layers in order to determine the update for the weights leading to the units in a hidden layer. The error at the output layer itself is computed using the difference between the desired output and the actual output at each of the output units. The actual output for a given input training pattern is determined by computing the outputs of units for each hidden layer in the forward pass of the input data. The error in the output is propagated backwards only to determine the weight updates [6].

\section{Methodology}

A network is to be designed and trained to recognize the 26 letters of the alphabet. An imaging system that digitizes each letter centered in the system's field of vision is available. The result is that each letter is represented as a 5 by 7 grid of boolean values [13].

For example, here is the letter A.

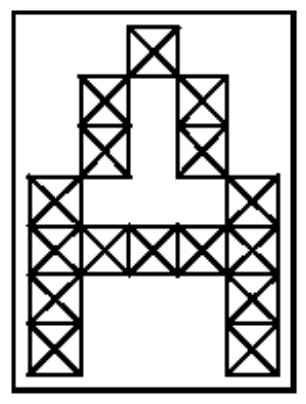

Figure: Letter 'A'

However, the imaging system is not perfect and the letters may suffer from noise. 


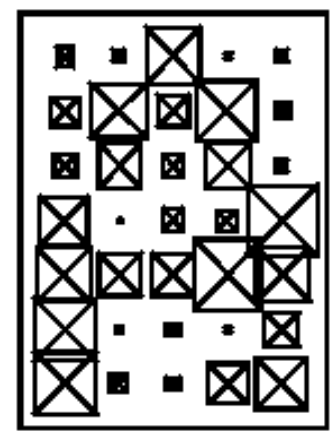

Figure: Noisy Characters 'A'

Perfect classification of ideal input vectors is required and reasonably accurate classification of noisy vectors. The twenty-six 35-element input vectors are defined in the function prprob as a matrix of input vectors called alphabet. The target vectors are also defined in this file with variable called targets. Each target vector is a 26-element vector with a 1 in the position of the letter it represents, and 0's everywhere else. For example, the letter A is to be represented by a 1 in the first element (as $\mathrm{A}$ is the first letter of the alphabet), and 0's in elements two through twenty-six.

\section{SYSTEM PERFORMANCE}

The reliability of the neural network pattern recognition system is measured by setting the network with hundreds of input vectors with varying quantities of noise. The script file tests the network at various noise levels, and then graphs the percentage of network errors versus noise. Noise with a mean of 0 and a standard deviation from 0 to 0.5 is added to input vectors. At each noise level, 100 presentations of different noisy versions of each letter are made and the network's output is calculated. The output is then passed through the competitive transfer function so that only one of the 26 outputs (representing the letters of the alphabet), has a value of 1 . The number of erroneous classifications is then added and percentages are obtained.

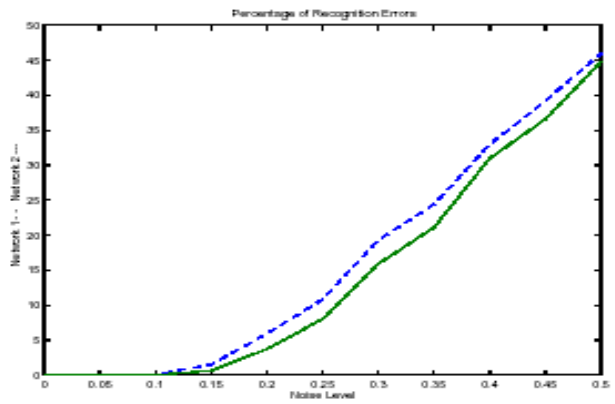

Graph: Shows the Reliability for the Network Trained with and without Noise

The solid line on the graph shows the reliability for the network trained with and without noise. The reliability of the same network when it had only been trained without noise is shown with a dashed line. Thus, training the network on noisy input vectors greatly reduces its errors when it has to classify noisy vectors. The network did not make any errors for vectors with noise of mean 0.00 or 0.05 . When noise of mean 0.2 was added to the vectors both networks began making errors. If a higher accuracy is needed, the network can be trained for a longer time or retrained with more neurons in its hidden layer. Also, the resolution of the input vectors can be 
increased to a 10-by-14 grid. Finally, the network could be trained on input vectors with greater amounts of noise if greater reliability were needed for higher levels of noise.

To test the system, a letter with noise can be created and presented to the network.

noisyJ = alphabet $(:, 10)+\operatorname{randn}(35,1) * 0.2$;

plotchar(noisyJ);

$\mathrm{A} 2=\operatorname{sim}($ net, noisy $\mathrm{J})$

$\mathrm{A} 2=\operatorname{compet}(\mathrm{A} 2)$;

answer $=$ find $(\operatorname{compet}(\mathrm{A} 2)==1)$;

plotchar(alphabet(:,answer));

In character below $\mathrm{J}$ is having $20 \%$ noise in scanning the character through scanning devise.

Here is the noisy letter and the letter the network picked (correctly).
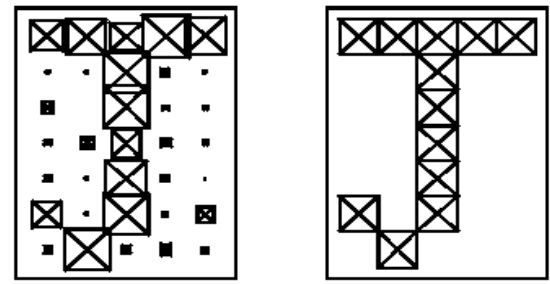

Figure: Noisy Letter ' $J$ ' and the Letter ' $J$ ' the Network Picked

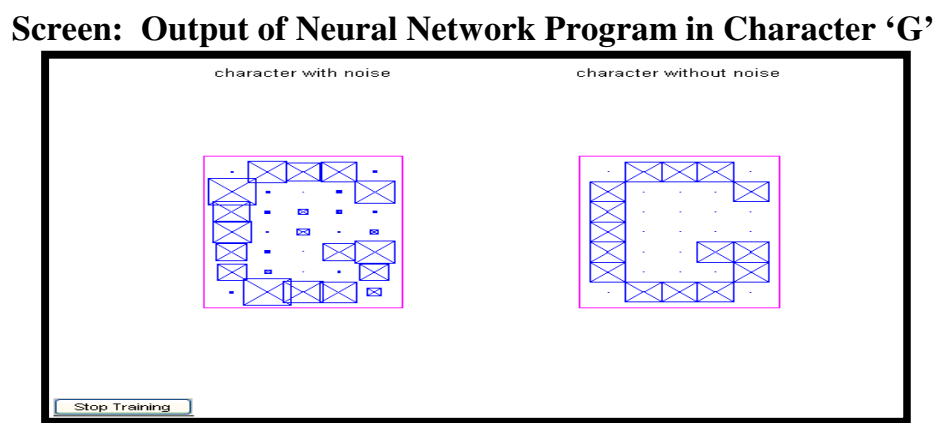

Following table shows percentage of noise in uppercase character ' $\mathrm{A}$ ' to ' $\mathrm{Z}$ ', serially or randomly:

Table: Percentage of Noise in Uppercase Character ' $A$ ' To ' $Z$ '

\begin{tabular}{|l|l|c|}
\hline Sr. no. & Character & $\begin{array}{l}\text { Max. percentage of noise } \\
\text { network picked (correctly) }\end{array}$ \\
\hline 1 & A & 88 \\
\hline 2 & B & 40 \\
\hline 3 & C & 45 \\
\hline 4 & D & 40 \\
\hline 5 & E & 45 \\
\hline 6 & F & 45 \\
\hline 7 & G & 50 \\
\hline 8 & H & 45 \\
\hline 9 & I & 40 \\
\hline 10 & J & 40 \\
\hline 11 & K & 50 \\
\hline 12 & L & 50 \\
\hline 13 & M & 35 \\
\hline
\end{tabular}


International Journal of Computer Science \& Engineering Survey (IJCSES) Vol.3, No.1, February 2012

\begin{tabular}{|l|l|l|}
\hline 14 & N & 40 \\
\hline 15 & O & 65 \\
\hline 16 & P & 60 \\
\hline 17 & Q & 65 \\
\hline 18 & R & 50 \\
\hline 19 & S & 50 \\
\hline 20 & T & 40 \\
\hline 21 & U & 55 \\
\hline 22 & V & 55 \\
\hline 23 & W & 60 \\
\hline 24 & X & 50 \\
\hline 25 & Y & 50 \\
\hline 26 & Z & 55 \\
\hline
\end{tabular}

The above table shows that almost all characters are recognized by the program. The character image contain $40 \%$ to $55 \%$ amount of noise in it.

Table: Comparison of Results by Computational and Analytical Method

Following graph shows graphical representation of numeric data of noise contained in the uppercase characters from ' $\mathrm{A}$ ' to $\mathrm{Z}$ ' which containing the 45 to $50 \%$ of noise.

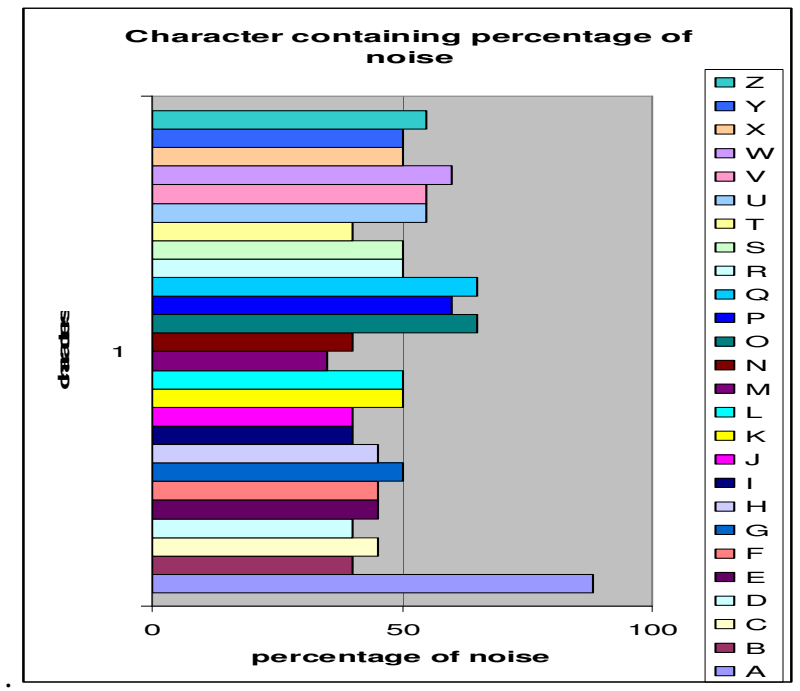

Graph: Characters Containing the Percentage of Noise

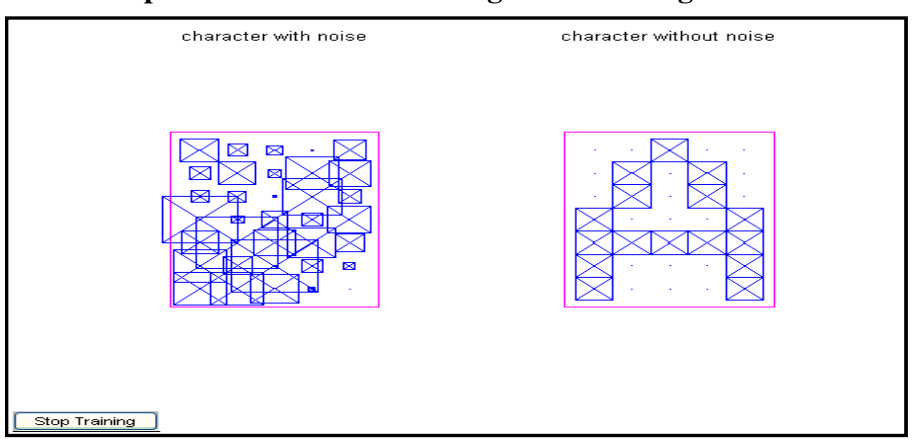

Screen:Character Containing $88 \%$ Noise in ' $A$ ' 
International Journal of Computer Science \& Engineering Survey (IJCSES) Vol.3, No.1, February 2012

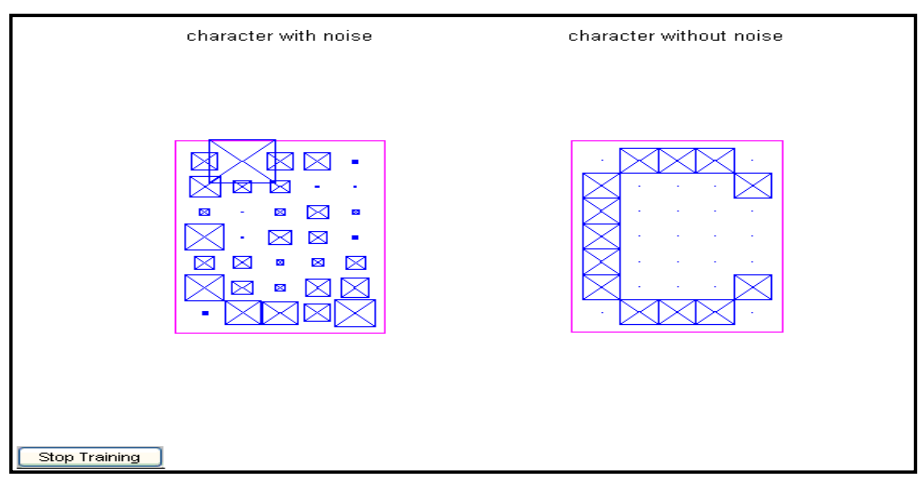

Screen:Character Containing $88 \%$ Noise in ' $\mathrm{A}$ '

\begin{tabular}{|c|c|c|c|c|}
\hline $\begin{array}{l}\text { Sr. } \\
\text { no. }\end{array}$ & Character & \multicolumn{2}{|c|}{$\begin{array}{l}\text { Max. Percentage of } \\
\text { noise network picked } \\
\text { character correctly. }\end{array}$} & $\begin{array}{l}\text { Noisy characters in } \\
\text { conventional } \\
\text { scanning method } \\
\text { in devise.(Omni - } \\
\text { scan) }\end{array}$ \\
\hline 1 & A & A & 88 & V \\
\hline 2 & B & B & 40 & $\mathrm{O}$ \\
\hline 3 & $\mathrm{C}$ & $\mathrm{C}$ & 45 & $\mathrm{O}$ \\
\hline 4 & $\mathrm{D}$ & $\mathrm{D}$ & 40 & Q \\
\hline 5 & $\mathrm{E}$ & $\mathrm{E}$ & 45 & $\mathrm{~T}$ \\
\hline 6 & $\mathrm{~F}$ & $\mathrm{~F}$ & 45 & $\mathrm{~T}$ \\
\hline 7 & $\mathrm{G}$ & $\bar{G}$ & 50 & $\mathrm{O}$ \\
\hline 8 & $\mathrm{H}$ & $\mathrm{H}$ & 45 & $\mathrm{M}$ \\
\hline 9 & $\mathrm{I}$ & $\mathrm{I}$ & 40 & 1 \\
\hline 10 & $\mathrm{~J}$ & $\mathrm{~J}$ & 40 & $\mathrm{I}$ \\
\hline 11 & $\mathrm{~K}$ & $\mathrm{~K}$ & 50 & $\mathrm{Y}$ \\
\hline 12 & $\mathrm{~L}$ & $\mathrm{~L}$ & 50 & I \\
\hline 13 & $\mathrm{M}$ & M & 35 & $\mathrm{M}$ \\
\hline 14 & $\mathrm{~N}$ & $\mathrm{~N}$ & 40 & $\mathrm{~N}$ \\
\hline 15 & $\mathrm{O}$ & $\mathrm{O}$ & 65 & $\mathrm{Q}$ \\
\hline 16 & $\mathrm{P}$ & $\mathrm{P}$ & 60 & $\mathrm{R}$ \\
\hline 17 & $\mathrm{Q}$ & Q & 65 & $\mathrm{O}$ \\
\hline 18 & $\mathrm{R}$ & $\mathrm{R}$ & 50 & $\mathrm{P}$ \\
\hline 19 & $S$ & $S$ & 50 & $\mathrm{~S}$ \\
\hline 20 & $\mathrm{~T}$ & $\mathrm{~T}$ & 40 & $\mathrm{I}$ \\
\hline 21 & $\mathrm{U}$ & $\mathrm{U}$ & 55 & $\mathrm{~V}$ \\
\hline 22 & $\mathrm{~V}$ & $\mathrm{~V}$ & 55 & $\mathrm{U}$ \\
\hline 23 & $\mathrm{~W}$ & $\mathrm{~W}$ & 60 & $\mathrm{~W}$ \\
\hline 24 & $\mathrm{X}$ & $\mathrm{X}$ & 50 & $\mathrm{Y}$ \\
\hline 25 & $\mathrm{Y}$ & $\mathrm{Y}$ & 50 & $\mathrm{X}$ \\
\hline 26 & $\mathrm{Z}$ & $\mathrm{Z}$ & 55 & 2 \\
\hline
\end{tabular}




\section{Training output of characters}

During experimentation it has been observed that Character Containing $88 \%$ Noise in letter ' $A$ ' recognized the character ' $A$ '. The letter ' $A$ ' is almost invisible for identification. Character containing $45 \%$ noise in ' $\mathrm{C}$ ', the letter ' $\mathrm{C}$ ' is still very difficult to recognize but recognized correctly the letter ' $C$ '. Character Containing 55\% Noise in letter ' $D$ ', looks very similar to letter ' $\mathrm{O}$ ', recognized correctly. Likewise all characters from ' $\mathrm{E}$ ' to ' $\mathrm{Z}$ ' recognized correctly.

\section{JUSTIFICATION OF DIFFERENCE}

The ANN is an information-processing paradigm inspired by the way the human brain processes information. Artificial neural networks are collections of mathematical models that represent some of the observed properties of biological nervous systems and draw on the analogies of adaptive biological learning. In today's network world, the need to maintain the security of information or physical property is becoming important and difficult. This technology is based in a field called "biometrics". Considering above Comparison of results obtained by simple conventional scanning system in scanning devise with the neural network technique in recognition of characters. Introducing the neural network method makes hundred percent perfect systems to identify the characters as compared to conventional scanning process in devise.

\section{Future scope}

In most of these crimes, the criminals were taking advantage of a fundamental flaw in the conventional access control systems. Now, technology will become available to allow verification of "true" individual identity. This technology is based in a field called "biometrics". The devises can be made intelligent by introducing the NN techniques can be embed the program into the hardware called "embedded system". The further scope of this can be in fingerprint identification, forensics, and signature verification/recognition of postage stamp amounts, handwritten text recognition, handwriting recognition language models, processing a handwritten sentence, document imaging or verification system, Arabic script recognition. Typewritten OCR, Handprint OCR, Cursive OCR Music OCR, Document imaging, It is check processing systems that use magnetic-ink character recognition (MICR). It is high speed check reader/sorter systems including microfilm, ink jet printer, and bar code reader features. Encoder printer, these are printers and their technologies that are available for the check and remittance applications.

\section{CONCLUSION}

Pattern association using back propagation algorithm is essential and helpful to optimize the association of input pattern to output pattern in the neural network. This approach can be used for pattern classification.

\section{REFERENCES}

[ 1] Ear Gose, Richard Johnsonsaugh, Steve Jost "Pattern Recognition and Image Processing", PHI, pp. 241-252.

[ 2] Duda, Hart, "Pattern Classification", TMH, pp. 13-24.

[ 3] Gonzalez and Woods, "Digital Image Processing", TMH, 2nd Edition, pp. 16-17. 
International Journal of Computer Science \& Engineering Survey (IJCSES) Vol.3, No.1, February 2012

[ 4] Raphael Feraud, "A Fast and Accurate Face Detector Based on Neural Networks", IEEE, Pattern Analysis and Machine Intelligence, Vol. 23, No.1, Jan 2001..

[5] Website: http://www.cedar.com/futureprospects.html

[6] Blanz and Vetter, "Face Recognition Based on Fitting a 3D Morphable Model", IEEE, Transactions on Pattern Analysis and Machine Intelligence, Vol. 25, No. 9, September 2003,pp. 1-5.

[ 7] B Yegnanarayana, “Artificial Neural Networks", PHI, 1999, pp. 16-17.

[ 8] Satish Kumar, "Neural Networks - A Classroom Approach", TMH, pp. 23-24. 